OPEN ACCESS

Edited by:

Bianca Weinstock-Guttman, University at Buffalo, United States

Reviewed by:

Murali Ramanathan,

SUNY Korea, South Korea Xiang Cao,

Nanjing Drum Tower Hospital, China

Juan Mei Zhang,

Nanjing University, China Hui Zhao,

Nanjing Drum Tower Hospital, China

*Correspondence:

Xuemei Chen

13347808579@189.cn

†These authors have contributed equally to this work

Specialty section:

This article was submitted to

Neuroepidemiology,

a section of the journal

Frontiers in Neurology

Received: 12 September 2020

Accepted: 12 February 2021

Published: 08 March 2021

Citation:

Jiang J, Gao Y, Zhang R, Wang L. Zhao X, Dai Q, Zhang W, Xu X and Chen $X$ (2021) Differential Effects of

Serum Lipoprotein-Associated Phospholipase A2 on Periventricular and Deep Subcortical White Matter

Hyperintensity in Brain.

Front. Neurol. 12:605372.

doi: 10.3389/fneur.2021.605372

\section{Differential Effects of Serum Lipoprotein-Associated Phospholipase A2 on Periventricular and Deep Subcortical White Matter Hyperintensity in Brain}

\author{
Junying Jiang ${ }^{1 \dagger}$, Yuanyuan Gao ${ }^{2 \dagger}$, Rui Zhang ${ }^{1}$, Lin Wang ${ }^{1}$, Xiaoyuan Zhao ${ }^{1}$, Qi Dai ${ }^{1}$, \\ Wei Zhang ${ }^{1}$, Xiujian $X^{1}{ }^{1}$ and Xuemei Chen ${ }^{1 *}$ \\ ${ }^{1}$ Department of Neurology, The Affiliated Jiangning Hospital With Nanjing Medical University, Nanjing, China, ${ }^{2}$ Department of \\ General Practice, The Affiliated Jiangning Hospital With Nanjing Medical University, Nanjing, China
}

Background and Purpose: Serum level of lipoprotein-associated phospholipase A2 (Lp-PLA2) was associated with white matter hyperintensity (WMH). There were differences in the anatomical structure and pathophysiological mechanism between periventricular WMH (PWWMH) and deep subcortical WMH (DSWMH). In this study, we aimed to investigate the effects of serum Lp-PLA2 on the PVWMH and DSWMH.

Methods: In total, 711 Chinese adults aged $\geq 45$ years with cranial magnetic resonance imaging (MRI) were recruited in this cross-sectional study, who had received physical examinations in the Department of Neurology, the Affiliated Jiangning Hospital of Nanjing Medical University due to dizziness and headaches between January 2016 and July 2019. Enzyme linked immunosorbent assay (ELISA) was utilized to determine the serum Lp-PLA2. Fazekas scale was used to measure the severity of PWWMH (grade 0-3) and DSWMH (grade 0-3) on MRI scans. Ordinal regression analysis was carried out to investigate the relationship between serum Lp-PLA2 and PWWMH or DSWMH.

Results: Finally, 567 cases were included in this study. The average level of serum Lp-PLA2 was $213.35 \pm 59.34 \mathrm{ng} / \mathrm{ml}$. There were statistical differences in the age, hypertension, diabetes mellitus, atrial fibrillation, lacunar infarction, Lp-PLA2 grade, creatinine, Hcy, and H-CRP $(P<0.05)$ in PVWMH groups. Ordinal regression analysis indicated that there was a lower risk of $\mathrm{PVWMH}$ in the patients with normal and moderately elevated serum Lp-PLA2 compared with those with significantly elevated serum Lp-PLA2 after adjusting age, hypertension, diabetes mellitus, atrial fibrillation, lacunar infarction, $\mathrm{Cr}$, Hcy, and H-CRP. In addition, PVWMH was correlated to advanced age, hypertension, diabetes mellitus, and lacunar infarction. After adjusting for confounding factors, DSWMH was correlated to advanced age and lacunar infarction. There was no correlation between serum Lp-PLA2 and DSWMH. 
Conclusions: Serum Lp-PLA2 was closely associated with the pathogenesis of $\mathrm{PWWMH}$ rather than DSWMH. There might be different pathological mechanisms between $\mathrm{PWWMH}$ and DSWMH.

Keywords: lipoprotein-associated phospholipase A2, periventricular white matter hyperintensity, deep subcortical white matter hyperintensity, Fazekas score, pathogenesis of PVWMH

\section{INTRODUCTION}

White matter hyperintensity (WMH), one the most common types of cerebral small vessel disease (CSVD) $(1,2)$, is mainly diagnosed based on the presence of hyperintensity on T2-weighted sequences, isointensity or hypointensity on T1weighted signals unlike cerebrospinal fluid. It depends on the sequence parameters and the severity of pathological changes (3). To date, WMH shows a high prevalence in the aged population (4-6), which affects stroke outcome (7-9), cognitive impairment $(10,11)$, and several neurological symptoms $(12,13)$. Therefore, it is necessary to screen the risk factors associated with the pathogenesis of $\mathrm{WMH}$.

Previous studies confirmed a relationship between inflammation and pathogenesis of WMH (14-16). Recently, a prospective study involving 15,792 adults aged between 45 and 65 years, midlife inflammation may lead to deterioration of $\mathrm{WMH}$ among the aged population (17). As an enzyme secreted by the circulating macrophages, lipoprotein-associated phospholipase A2 (Lp-PLA2) participated in the low density lipoprotein metabolism, contributed to the onset of atherosclerosis, and mediated the inflammation $(18,19)$. In a previous study, homocysteine (Hcy) concentration was associated with the increased burden of WMH (20). However, studies on the relationship between Lp-PLA2 and $\mathrm{WMH}$ are relatively few. According to the lesion sites, WMH could be divided into periventricular WMH (PVWMH) and deep subcortical WMH (DSWMH) (20). Generally, these two types of WMH are usually simultaneously developed and progressed. Nevertheless, increasing studies indicated that PVWMH and DSWMH showed various anatomical and histopathological changes, which demonstrated that there might be differences in their pathogenesis $(20,21)$. To date, no studies focused on the effects of serum Lp-PLA2 levels on the variations between PVWMH and DSWMH. In this study, we aimed to determine the impacts of serum Lp-PLA2 level on the onset of PVWMH and DSWMH.

\section{METHODS}

\section{Subjects}

In this study, we included 711 cases of patients who received physical examinations in the Department of Neurology, the Affiliated Jiangning Hospital of Nanjing Medical University due to dizziness and headaches between January 2016 and July 2019. Finally, 567 subjects met the inclusion and exclusion criteria. The inclusion criteria were as follows: those aged $\geq 45$ years; those had received cerebral MRI. The exclusion criteria were as follows: those without Lp-PLA2 data, those with acute or chronic infectious diseases or rheumatic diseases, or those that may affect the evaluation of $\mathrm{WMH}$, those with severe head injury, severe cerebral infarction, cerebral hemorrhage, multiple sclerosis, or brain malignancy. The study flowchart was shown in Figure 1. Each subject signed the informed consent. The study protocols were approved by the Ethical Committee of Affiliated Jiangning Hospital of Nanjing Medical University.

\section{Data Collection}

The risk factors for cardiovascular diseases were collected from each subject, including clinical and demographic information. The information consisted of age, gender, hypertension, diabetes mellitus, coronary heart disease, atrial fibrillation, lacunar infarction, smoking, drinking alcohol, as well as formation of carotid artery plaque. In addition, laboratory tests were carried out to determine the fasting blood glucose (FBG), total cholesterol (TC), triglyceride (TG), low density lipoprotein (LDL), Hcy, Lp-PLA2, high-sensitive C-reactive protein ( $\mathrm{H}$ $\mathrm{CRP})$, blood urea nitrogen (BUN), as well as creatinine (Cr). All the samples were collected from the venous blood of the subjects in a fasting state for $12 \mathrm{~h}$. The serum Lp-PLA2 concentration was determined using the ELISA method, and was divided into normal range $(<200 \mu \mathrm{g} / \mathrm{L})$, moderate elevation (200-223 $\mu \mathrm{g} / \mathrm{L}$ ), and significant elevation ( $\geq 223 \mu \mathrm{g} / \mathrm{L}$ ) according to the 2012 AACE' Guidelines for Management of Dyslipidemia and Prevention of Atherosclerosis (22). Immunoturbidimetry was used to determine the level of H-CRP, and the concentration of Hcy was determined using the enzymatic method. The diagnosis of carotid artery plaque was given based on the local intima media thickness (IMT) of $>1.2 \mathrm{~mm}$ or a 1.5 -fold or more to the peripheral IMT. The site for the quantification of IMT measurement was chosen in the area with a distance of about $1.5 \mathrm{~cm}$ to the carotid bifuracation (23).

\section{Cranial MRI Collection and WMH}

For the collection of cranial MRI, the MRI was performed using a 3.0T system, equipped with 8-channel coil arrays (Ingenia, Philips Medical System). MRI consisted of T1-weighted image, T2-weighted image, FLAIR image, and DWI findings. For the $\mathrm{WMH}$, there was hyperintensity on T2-weighted images or FLAIR images in the lateral ventricle and subcortex region. $\mathrm{WMH}$ was confirmed in the presence of isointensity or hypointensity on T1-weighted signals (3). WHM was evaluated using the Fazekas scale (24). In the Fazekas scale, the lesions in the PVWMH and DSWMH were evaluated separately. Specifically, the PVWMH score standards were as follows: 0, loss; 1, calyptriform or pencil-like lamella; 2, smooth halo; 3 , irregular hypertense signals near the ventricle extending to the deep white matter. The DSWMH score standards were as follows: 0 , loss; 1 , punctiform lesions; 2 , initial fusion in the lesions; 3 , 


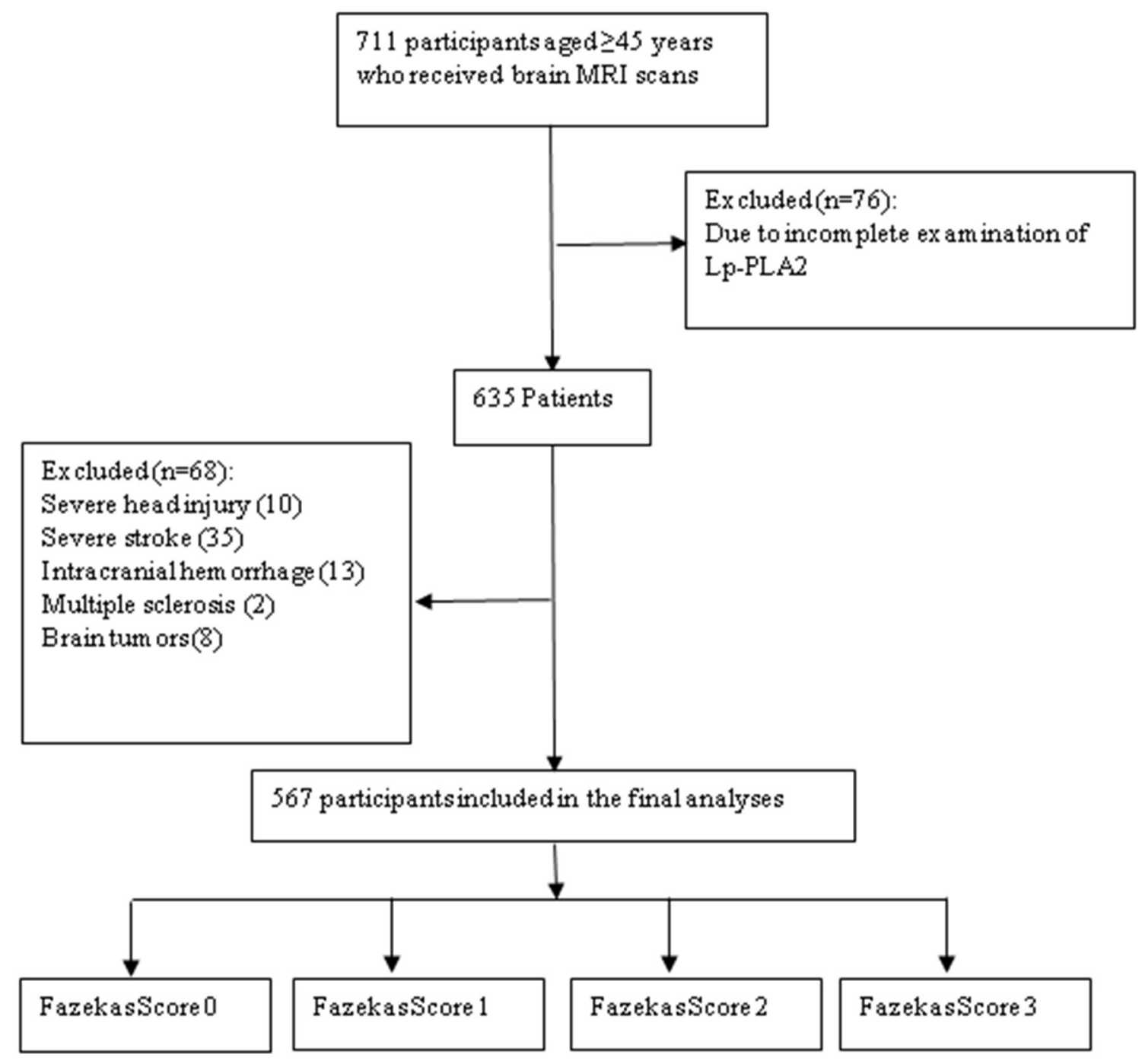

FIGURE 1 | Study flowchart.

massive fusion in the lesions (Figure 2). The Fazekas scale was evaluated by two experienced staff, specializing in radiation, who were blinded to the study, they reached a consensus through discussion to resolve their differences.

\section{Statistical Analysis}

All the continuous data were presented as mean \pm standard deviation, or the median (quartile). The categorical data were presented as the percentage. The continuous variables that were normally distributed were analyzed using the one-factor analysis of variance, while these that were not normally distributed were analyzed using the non-parametric test. The categorical variables were analyzed using the Chi square test. The independent risk factors for PVWMH and DSWMH were measured using the odds ratio (OR) or $95 \%$ confidential interval (95\% CI), based on the ordinal regression analysis. SPSS 21.0 software was utilized for the statistical analysis. $P<0.05$ was considered to be statistically significant.

\section{RESULTS}

\section{Characteristics of the Subjects}

In total, 711 subjects were enrolled at first, among which 567 (male: 293; female: 274; median age: $65.51 \pm 9.86$ years) were finally included in this study. All the 567 subjects received Fazekas score evaluation. Finally, 468 cases $(82.54 \%)$ were diagnosed with WMH, including 432 (76.19\%) with PVWMH and $378(66.66 \%)$ with DSWMH. Among these cases, 340 (59.96\%) showed serum Lp-PLA2 of <200 $\mu \mathrm{g} / \mathrm{L}, 26(4.59 \%)$ showed serum Lp-PLA2 in a range of $200-223 \mu \mathrm{g} / \mathrm{L}$, and the rest 201 (35.45\%) showed serum Lp-PLA2 of $\geq 223 \mu \mathrm{g} / \mathrm{L}$ (Table 1). 

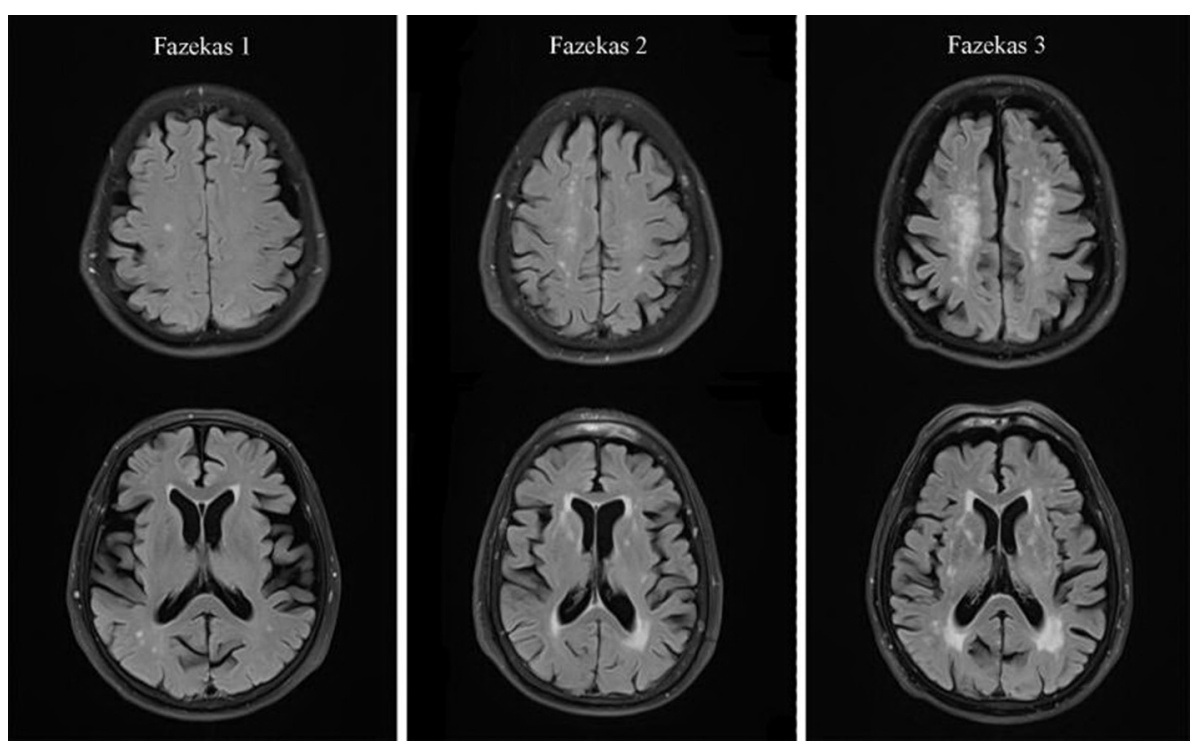

FIGURE 2 | Representative T2-FLAIR images illustrating Fazekas score.

TABLE 1 | Baseline characteristics of the cohort.

\begin{tabular}{lc}
\hline Clinical characteristics & Results \\
\hline Age, yr & $65.51 \pm 9.86$ \\
FBG, mmol/L & $5.95 \pm 2.12$ \\
TC, mmol/L & $4.41 \pm 1.05$ \\
TG, mmol/L & $2.20 \pm 9.62$ \\
LDL-C, mmol/L & $2.41 \pm 0.82$ \\
HDL-C, mmol/L & $1.21 \pm 0.34$ \\
H-CRP, mg/L & $0.34 \pm 0.08$ \\
BUN, mmol/L & $5.50 \pm 4.14$ \\
Cr, $\mu$ mol/L & $69.15 \pm 21.23$ \\
Hcy, $\mu$ mol/L & $12.00 \pm 7.80$ \\
Sex, men & $51.68 \%$ \\
Hypertension, \% & $70.90 \%$ \\
Diabetes mellitus, \% & $23.28 \%$ \\
Coronary disease, \% & $9.70 \%$ \\
Atrial fibrillation, \% & $2.65 \%$ \\
Smoking, \% & $19.22 \%$ \\
Alcohol, \% & $12.00 \%$ \\
Carotid atherosclerosis, \% & $58.91 \%$ \\
WMH, \% & $82.54 \%$ \\
PWWMH, \% & $76.19 \%$ \\
DSWMH, \% & $66.66 \%$ \\
LI, \% & $49.74 \%$ \\
LP-PLA2 $\% 200$ ng/ml, \% & $59.96 \%$ \\
& \\
\hline & \\
\hline
\end{tabular}

WMH, white matter hyperintensity; PWWMH, periventricular WMH; DSWMH, deep subcortical WMH; LI, Lacunar infarction; FPG, fasting blood glucose; TC, total cholesterol; TG, triglyceride; LDL-C, low density lipoprotein cholesterol; HDL-C, high density lipoprotein cholesterol; BUN, blood urea nitrogen; $\mathrm{Cr}$, creatinine; $\mathrm{H}$-CRP, high C-reactive protein; Hcy, homocysteine; LP-PLA2, lipoprotein-associated phospholipase A2.

\section{One-Factor Analysis for PVWMH and DSWMH}

According to the PVWMH grouping, 135 subjects showed a Fazekas score of 0, 251 showed a score of 1, 107 showed a score of 2 , and 74 showed a score of 3 . There were statistical differences in the age, hypertension, diabetes mellitus, atrial fibrillation, lacunar infarction, Lp-PLA2 grade, creatinine, Hcy, and H-CRP $(P<0.05$, Table 2). For the DSWMH grouping, 189 subjects showed a Fazekas score of 0, 244 showed a score of 1 , 84 showed a score of 2 , and 50 showed a score of 3 . There were statistical differences in the age, hypertension, diabetes mellitus, lacunar infarction, Lp-PLA2 grade, TG, and Hcy $(P<0.05$, Table 3).

\section{Independent Correlation Factors for PVWMH}

To further evaluate the correlation factors for PVWMH and DSWMH, we established an ordinal regression model for the factors that may affect the PVWMH and DSWMH. Prior to the establishing of an ordinal regression model for PVWMH, we then performed the fitting test for the model $\left(\chi^{2}=261.40, P<0.001\right)$ and the linear hypothesis test $(\mathrm{P}=0.148)$, which indicated that the model showed good fitting. The ordinal regression analysis indicated that, after adjusting for age, hypertension, diabetes mellitus, atrial fibrillation, lacunar infarction, $\mathrm{Cr}$, Hcy, and $\mathrm{H}-$ CRP, there was a lower risk of PVWMH in the patients with normal and moderately elevated serum Lp-PLA2 compared with those with significantly elevated serum Lp-PLA2 (normal LpPLA2: OR 0.258, 95\% CI 0.119-0.520; moderate Lp-PLA2: OR $0.305,95 \%$ CI $0.134-0.696$ ) after adjusting for the confounding variables. In addition, PVWMH grade was positively correlated 
TABLE 2 | Clinical characteristics of subjects with PVWMH.

\begin{tabular}{|c|c|c|c|c|c|}
\hline Variable & $\begin{array}{l}\text { Fazekas grade } 0 \\
\quad(n=135)\end{array}$ & $\begin{array}{l}\text { Fazekas grade } 1 \\
(n=251)\end{array}$ & $\begin{array}{c}\text { Fazekas grade } 2 \\
\quad(n=107)\end{array}$ & $\begin{array}{c}\text { Fazekas grade } 3 \\
\quad(n=74)\end{array}$ & $P$-value \\
\hline Age, yr & $59.83 \pm 9.22$ & $64.06 \pm 8.98$ & $70.44 \pm 7.74$ & $73.65 \pm 8.01$ & $<0.01$ \\
\hline Hypertension, n (\%) & $71(52.59)$ & $179(71.31)$ & 86(80.37) & 66(89.19) & $<0.01$ \\
\hline Diabetes mellitus, n (\%) & $17(12.59)$ & $58(23.11)$ & $34(31.78)$ & 23(31.08) & $<0.01$ \\
\hline LI, $n(\%)$ & 19(14.07) & $122(48.61)$ & $74(69.16)$ & $67(90.54)$ & $<0.01$ \\
\hline Smoking, $n(\%)$ & $27(20.00)$ & $44(17.53)$ & $22(20.56)$ & 16(21.62) & 0.822 \\
\hline Alcohol, $n$ (\%) & $20(14.81)$ & 25(9.96) & $15(14.02)$ & $8(10.81)$ & 0.473 \\
\hline \multicolumn{6}{|l|}{ LP-PLA2 } \\
\hline$<200 \mathrm{ng} / \mathrm{ml}, n(\%)$ & $41(30.37)$ & $57(22.71)$ & $30(28.04)$ & $12(16.22)$ & 0.007 \\
\hline TG, median (IQR) & $1.43(1.03-2.10)$ & $1.58(1.09-2.17)$ & $1.34(1.06-1.76)$ & $1.53(1.02-2.24)$ & 0.101 \\
\hline LDL-C, median (IQR) & $2.46(1.96-2.98)$ & $2.38(1.84-2.96)$ & $2.25(1.73-2.79)$ & $2.27(1.84-2.81)$ & 0.465 \\
\hline HDL-C, median (IQR) & $1.17(1.00-1.38)$ & $1.18(0.99-1.35)$ & $1.18(0.96-1.44)$ & $1.10(0.97-1.39)$ & 0.643 \\
\hline BUN, median (IQR) & $5.02(4.02-5.90)$ & 4.99(4.08-6.10) & $5.10(4.11-6.40)$ & $5.18(4.11-6.21)$ & 0.526 \\
\hline $\mathrm{Cr}$, median (IQR) & $63.00(53.00-79.40)$ & $65.00(55.00-77.00)$ & $63.00(54.00-78.00)$ & 70.50(62.98-86.13) & 0.003 \\
\hline Hcy (IQR) & $10.10(7.10-12.80)$ & 10.40(7.93-13.20) & 11.60(8.80-15.60) & 12.65(9.80-15.58) & $<0.01$ \\
\hline H-CRP, median (IQR) & $0.20(0.12-0.25)$ & $0.30(0.24-0.35)$ & $0.50(0.25-0.60)$ & $0.60(0.34-0.70)$ & 0.004 \\
\hline
\end{tabular}

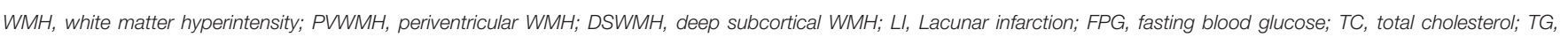

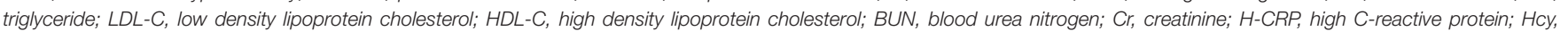
homocysteine; LP-PLA2, lipoprotein-associated phospholipase A2.

with the advanced age (OR 1.089, 95\% CI 1.067-1.110), hypertension (OR 1.614, 95\% CI 1.105-2.358), diabetes mellitus $(\mathrm{OR}=1.579,95 \% \mathrm{CI} 1.078-2.314)$, as well as lacunar infarction (OR 5.155, 95\% CI 3.540-7.508, Table 4).

\section{Independent Correlation Factors for DSWMH}

After fitting test and linear hypothesis test, the ordinal regression analysis indicated a good fitting for the DSWMH (fitting test: $\chi^{2}=198.546, P<0.001$; linear hypothesis test: $\left.P=0.136\right)$. The ordinal regression analysis indicated that, after adjusting for age, hypertension, diabetes mellitus, lacunar infarction, Cr, TG, and Hcy, there was a positive correlation between DSWMH and advanced age (OR 1.081, 95\% CI 1.061-1.102) and lacunar infarction (OR 3.823, 95\% CI 2.662-5.490, Table 5).

\section{DISCUSSION}

Rare studies investigated the correlation between serum LpPLA2 and WMH. Previously, Lp-PLA2 was reported to associate with the WMH $(25,26)$. However, there are nearly no studies which have been conducted to illustrate the roles of serum LpPLA2 on the sites of WMH. In this study, we investigated the effects of serum Lp-PLA2 on PVWMH and DSWMH in the elder population. Ordinal regression analysis indicated that the patients with normal and moderately elevated serum Lp-PLA2 showed lower risks of developing PVWMH compared with those with significantly elevated serum Lp-PLA2. Our data revealed that statistical differences were merely observed between the patients with moderately and significantly elevated Lp-PLA2, which may be related to the fact that the sample size was not large. Moreover, no correlation was noticed between serum LpPLA2 and DSWMH. The serum Lp-PLA2 was independently correlated to the PVWMH rather than DSWMH. These findings indicated that Lp-PLA2 metabolic disorder played a crucial role in the pathogenesis of PVWMH.

$\mathrm{WMH}$ is featured by demyelination, loss of axon and gliosis (27). To date, there are still disputes on the pathogenesis of WMH. Inflammation was considered to be associated with the pathogenesis of $\mathrm{WMH}$, however, in some circumstances, inflammation is a biological response to the infection and injury (28). Previously, inflammation was well-accepted to be closely related to the pathogenesis of $\mathrm{WMH}$ (14-16). In addition, there were regional variations in the effects of inflammation on the WMH. Inflammation was correlated with PVWMH rather than DSWMH (28), which was in line with our data. Compared with systemic inflammation biomarkers, there was a stronger correlation between vascular inflammation markers and WMH (28). In addition, uni-variate analysis indicated a 
TABLE 3 | Clinical characteristics of subjects with DSWMH.

\begin{tabular}{|c|c|c|c|c|c|}
\hline Variable & $\begin{array}{l}\text { Fazekas grade } 0 \\
\quad(n=189)\end{array}$ & $\begin{array}{l}\text { Fazekas grade } 1 \\
\quad(n=244)\end{array}$ & $\begin{array}{c}\text { Fazekas grade } 2 \\
(n=84)\end{array}$ & $\begin{array}{l}\text { Fazekas grade } 3 \\
\quad(n=50)\end{array}$ & $P$-value \\
\hline Age, yr & $60.40 \pm 9.13$ & $66.17 \pm 8.93$ & $69.30 \pm 8.71$ & $75.20 \pm 7.42$ & $<0.01$ \\
\hline Hypertension, $n(\%)$ & 112(59.26) & 173(70.90) & $73(86.90)$ & $44(88.00)$ & $<0.01$ \\
\hline Diabetes mellitus, $n(\%)$ & $34(17.99)$ & $54(22.13)$ & $30(35.71)$ & $14(28.00)$ & 0.012 \\
\hline LI, $n(\%)$ & $46(24.34)$ & $130(53.28)$ & 62(73.81) & $44(88.00)$ & $<0.01$ \\
\hline Smoking, $n(\%)$ & $44(23.28)$ & $38(15.57)$ & $18(21.43)$ & $9(18.00)$ & 0.221 \\
\hline Alcohol, $n(\%)$ & $26(13.76)$ & $25(10.25)$ & $10(11.90)$ & $7(14.00)$ & 0.693 \\
\hline \multicolumn{6}{|l|}{ LP-PLA2 } \\
\hline$<200 \mathrm{ng} / \mathrm{ml}, n(\%)$ & 59(31.22) & $53(21.72)$ & $25(29.76)$ & $13(26.00)$ & 0.038 \\
\hline TG, median (IQR) & $1.43(1.03-2.08)$ & $1.56(1.18-2.30)$ & $1.34(1.00-1.82)$ & $1.55(0.93-2.05)$ & 0.038 \\
\hline LDL-C, median (IQR) & $2.45(1.95-3.03)$ & $2.36(1.87-2.87)$ & $2.16(1.53-2.64)$ & $2.48(1.75-2.96)$ & 0.052 \\
\hline HDL-C, median (IQR) & $1.17(0.97-1.40)$ & $1.20(1.00-1.38)$ & $1.15(0.95-1.35)$ & $1.17(0.97-1.48)$ & 0.747 \\
\hline BUN, median (IQR) & $5.01(4.10-5.90)$ & $5.11(4.08-6.12)$ & $4.77(3.93-6.22)$ & $5.21(4.42-6.24)$ & 0.623 \\
\hline $\mathrm{Cr}$, median (IQR) & $63.00(53.55-77.15)$ & $65.00(56.00-77.93)$ & $65.45(55.25-81.23)$ & $70.50(60.35-88.03)$ & 0.058 \\
\hline Hcy (IQR) & $10.40(7.50-13.20)$ & $10.40(7.93-14.28)$ & 11.80(8.63-16.03) & 12.60(9.98-15.10) & 0.003 \\
\hline H-CRP, median (IQR) & $0.20(0.15-0.25)$ & $0.30(0.24-0.35)$ & $0.42(0.30-0.46)$ & $0.45(0.28-0.48)$ & 0.223 \\
\hline
\end{tabular}

WMH, white matter hyperintensity; PWWMH, periventricular WMH; DSWMH, deep subcortical WMH; LI, Lacunar infarction; FPG, fasting blood glucose; TC, total cholesterol; TG, triglyceride; LDL-C, low density lipoprotein cholesterol; HDL-C, high density lipoprotein cholesterol; BUN, blood urea nitrogen; Cr, creatinine; H-CRP, high C-reactive protein; Hcy, homocysteine; LP-PLA2, lipoprotein-associated phospholipase A2.

TABLE 4 | Ordinal regression model analysis of influencing factors of PWWMH classifications.

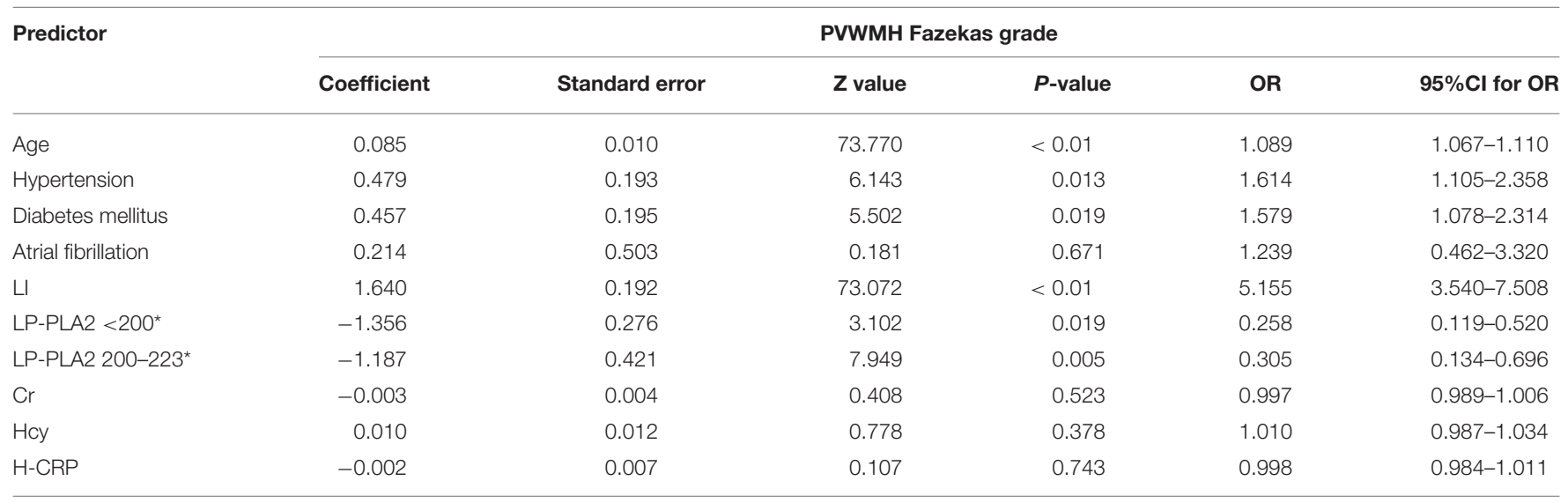

LI, lacunar infarction; Cr, creatinine; H-CRP, high C-reactive protein; Hcy, homocysteine; Lp-PLA2, lipoprotein-associated phospholipase A2; *LP-PLA2 >232.00 ug/L served as control.

correlation between H-CRP and PVWMH. After adjusting age, hypertension, diabetes mellitus, atrial fibrillation and lacunar infarction, creatinine, Hcy, and phosphatidase A2, there was no correlation between H-CRP and PVWMH. Similarly, there was no correlation between H-CRP and DSWMH. Nevertheless, systemic inflammation may contribute to the pathogenesis of $\mathrm{WMH}$ in the elder population in a recent prospective study involving a large sample size. This implied that systemic inflammation was correlated with the progression of CSVD, especially in the conditions with persistent inflammation (17). In future, further prospective cohort studies are required to illustrate the exact causes.

Lp-PLA2 is a type of enzyme involved in the hydrolysis of phosphoric oxide into oxidant fatty acids and lysophosphatidyl 
TABLE 5 | Ordinal regression model analysis of influencing factors of DSWMH classifications.

\begin{tabular}{|c|c|c|c|c|c|c|}
\hline \multirow[t]{2}{*}{ Predictor } & \multicolumn{6}{|c|}{ DSWMH Fazekas grade } \\
\hline & Coefficient & Standard error & $Z$-value & $P$-value & OR & $95 \% \mathrm{Cl}$ for OR \\
\hline Age & 0.078 & 0.010 & 66.428 & $<0.01$ & 1.081 & $1.061-1.102$ \\
\hline Hypertension & 0.348 & 0.194 & 3.221 & 0.073 & 1.419 & $0.969-2.071$ \\
\hline Diabetes mellitus & 0.272 & 0.192 & 1.996 & 0.158 & 1.313 & $0.900-1.912$ \\
\hline LI & 1.341 & 0.185 & 52.774 & $<0.01$ & 3.823 & $2.662-5.490$ \\
\hline LP-PLA2 <200* & -0.102 & 0.174 & 0.344 & 0.557 & 0.903 & $0.641-1.271$ \\
\hline LP-PLA2 200-223* & -0.423 & 0.410 & 1.063 & 0.303 & 0.655 & $0.293-1.464$ \\
\hline TG & 0.027 & 0.025 & 1.109 & 0.292 & 1.027 & $0.977-1.080$ \\
\hline Hcy & -0.000 & 0.011 & 0.002 & 0.969 & 1 & $0.979-1.020$ \\
\hline
\end{tabular}

LI, lacunar infarction; TG, triglyceride; Hcy, homocysteine; LP-PLA2, lipoprotein-associated phospholipase A2; ${ }^{*} L P-P L A 2>232.00$ ug/L served as control.

choline $(23,25)$. It is closely related to ischemic stroke (29) or vascular dementia (30). Besides, it contributes to the pathogenesis of atherosclerosis (31) and mediation of inflammation $(29,32)$. Moreover, it can mediate the vasculitis through regulating the metabolism of blood fat (33). To our best knowledge, few studies have been focused on the roles of Lp-PLA2 in the WMH. In a cross-sectional study involving 527 stroke-free subjects, Wright et al. indicated that Lp-PLA2 was associated with a greater burden of WMH independent of H-CRP. However, that study did not investigate the potential effects of Lp-PLA2 on the WMH in different regions (19). Our study indicated a correlation between elevation of serum Lp-PLA2 and PVWMH rather than DSWMH.

As is known to all, PVWMH usually occurs simultaneously with DSWMH. With the aging process, WMH usually happens in a single region of the white matter, which then progresses to another region, such as from the periventricular region to the deep subcortical region (20). Our data indicated that serum Lp-PLA2 showed various effects on PVWMH and DSWMH, however, we could not explain the exact mechanism and causes. To date, little is known about why inflammation induces various effects on PVWMH or DSWMH. In the previous studies $(3,21), \mathrm{PVWMH}$ and DSWMH were considered to reflect various anatomical and histopathological features. In the anatomical view, there were anatomical differences between the arteriole in the peripheral ventricle and the deep subcortical region, however, the periventricular arteriola was superior to the deep subcortical arteriola in protecting the peripheral tissues from the vascular factors. In the histopathological views, DSWMH was likely to present more ischemic injuries, while PVWMH may involve more inflammatory metabolism (3436). The inflammatory cascade reaction would trigger the endothelial dysfunction, which subsequently led to a slight dysfunction of the blood-brain barrier and $\mathrm{WMH}$ caused by tissue damages $(27,37)$. The calyptriform or smooth halo lesions in the peripheral ventricle were not originated from the ischemia. In fact, it was the subendymal gliosis and the demyelinated region beneath the ependyma lining. The deep subcortical punctiform signals, early fusion and fused white matter hypointensity indicated the gradual increase of the tissues with ischemic injuries, which was featured by slight perivascular lesions to massive axon loss, multiple small vacuoles and obvious arteriolosclerosis $(34-36,38)$. Therefore, these differences were tended to support the fact that serum Lp-PLA2 was more closely correlated to PVWMH rather than DSWMH.

Indeed, there are some limitations in this study. Firstly, we could not obtain a causal speculation based on these crosssectional studies. Secondly, we only analyzed the circulating biomarkers at a certain time point. Therefore, prospective and longitudinal studies are required to illustrate the causal relationship between Lp-PLA2 and WMH. Thirdly, the Fazekas score was utilized for the grading of the $\mathrm{WMH}$, without performing the quantification analysis. Fourth, more basic research is needed to further explore the pathogenesis of its differential effects.

\section{CONCLUSIONS}

Serum Lp-PLA2 was closely related to the pathogenesis of PVWMH rather than DSWMH. Our data indicated that there might be different pathological mechanisms between PVWMH and DSWMH. In addition, Lp-PLA2 played an important role in the pathogenesis and progression of PVWMH.

\section{DATA AVAILABILITY STATEMENT}

The raw data supporting the conclusions of this article will be made available by the authors, without undue reservation.

\section{ETHICS STATEMENT}

The studies involving human participants were reviewed and approved by Medical ethics committee of Affiliated Drum Tower Hospital of Nanjing University Medical School (2016YFC1300500). The patients/participants provided their written informed consent to participate in this study. Written informed consent was obtained from the individual(s) for the publication of any potentially identifiable images or data included in this article. 


\section{AUTHOR CONTRIBUTIONS}

JJ and YG: design of the study. RZ, LW, XZ, WZ, QD, and XX: collection of data. YG and JJ: statistical analysis. JJ, YG, and $\mathrm{XC}$ : writing-review and editing of the drafts. All authors had full access to all the data in the study and take responsibility of the data and the accuracy of the data analysis.

\section{FUNDING}

This research was supported by Kangda College of Nanjing Medical University Science and Research

\section{REFERENCES}

1. Soldan A, Pettigrew C, Zhu Y, Wang MC, Moghekar A, Gottesman RF, et al. White matter hyperintensities and CSF Alzheimer disease biomarkers in preclinical Alzheimer disease. Neurology. (2020) 94:e950-e60. doi: 10.1212/WNL.0000000000008864

2. Rensma SP, van Sloten TT, Launer LJ, Stehouwer CDA. Cerebral small vessel disease and risk of incident stroke, dementia and depression, and all-cause mortality: A systematic review and meta-analysis. Neurosci Biobehav Rev. (2018) 90:164-73. doi: 10.1016/j.neubiorev.2018.04.003

3. Wardlaw JM, Smith EE, Biessels GJ, Cordonnier C, Fazekas F, Frayne R, et al. Neuroimaging standards for research into small vessel disease and its contribution to ageing and neurodegeneration. Lancet Neurol. (2013) 12:822-38. doi: 10.1016/S1474-4422(13)70124-8

4. T PS, Srikanth VK, Hubbard RE, Moran C, Beare R, Wood A, et al. White matter hyperintensities and the progression of frailty-the Tasmanian Study of Cognition and Gait. J Gerontol A Biol Sci Med Sci. (2020) 75:1545-50. doi: 10.1093/gerona/glaa024

5. Zhou YN, Gao HY, Zhao FF, Liang YC, Gao Y, Liu XH, et al. The study on analysis of risk factors for severity of white matter lesions and its correlation with cerebral microbleeds in the elderly with lacunar infarction. Medicine (Baltimore). (2020) 99:e18865. doi: 10.1097/MD.0000000000 018865

6. Molloy CJ, Nugent S, Bokde ALW. Alterations in diffusion measures of white matter integrity associated with healthy aging. J Gerontol A Biol Sci Med Sci. (2019). doi: 10.1101/540443

7. Zerna C, Yu AYX, Modi J, Patel SK, Coulter JI, Smith EE, et al. Association of white matter hyperintensities with short-term outcomes in patients with minor cerebrovascular events. Stroke. (2018) 49:919-23. doi: 10.1161/STROKEAHA.117.017429

8. Georgakis MK, Duering $M$, Wardlaw JM, Dichgans $M$. WMH and long-term outcomes in ischemic stroke: a systematic review and metaanalysis. Neurology. (2019) 92:e1298-e308. doi: 10.1212/WNL.0000000000 007142

9. Debette S, Schilling S, Duperron MG, Larsson SC, Markus HS. Clinical Significance of magnetic resonance imaging markers of vascular brain injury: a systematic review and meta-analysis. JAMA Neurol. (2019) 76:81-94. doi: 10.1001/jamaneurol.2018.3122

10. Knopman DS, Griswold ME, Lirette ST, Gottesman RF, Kantarci K, Sharrett AR, et al. Vascular imaging abnormalities and cognition: mediation by cortical volume in nondemented individuals: atherosclerosis risk in communities-neurocognitive study. Stroke. (2015) 46:433-40. doi: 10.1161/STROKEAHA.114.007847

11. van den Berg E, Geerlings MI, Biessels GJ, Nederkoorn PJ, Kloppenborg RP. White matter hyperintensities and cognition in mild cognitive impairment and alzheimer's disease: a domain-specific meta-analysis. J Alzheimers Dis. (2018) 63:515-27. doi: 10.3233/JAD-170573

12. Pinter D, Ritchie SJ, Doubal F, Gattringer T, Morris Z, Bastin ME, et al. Impact of small vessel disease in the brain on gait and balance. Sci Rep. (2017) 7:41637. doi: $10.1038 /$ srep41637
Development Project, Jiangsu, China (KD2019KYJJYB047); Nanjing Medical Science and Technique Development Foundation (QRX17032); Nanjing Medical University Science and Technology Development Project, Jiangsu, China (NMUB2019240).

\section{ACKNOWLEDGMENTS}

This manuscript has been released as a pre-print at Research Square, 27 July 2020, PREPRINT (Version 1) available at Research Square (doi: 10.21203/rs.3.rs-44790/v1) (39).
13. van Agtmaal MJM, Houben A, Pouwer F, Stehouwer CDA, Schram MT. Association of microvascular dysfunction with late-life depression: a systematic review and meta-analysis. JAMA Psychiatry. (2017) 74:729-39. doi: 10.1001/jamapsychiatry.2017.0984

14. Satizabal CL, Zhu YC, Mazoyer B, Dufouil C, Tzourio C. Circulating IL-6 and CRP are associated with MRI findings in the elderly: the 3C-Dijon Study. Neurology. (2012) 78:720-7. doi: 10.1212/WNL.0b013e318248e50f

15. Wersching $\mathrm{H}$, Duning $\mathrm{T}$, Lohmann $\mathrm{H}$, Mohammadi S, Stehling $\mathrm{C}$, Fobker $M$, et al. Serum C-reactive protein is linked to cerebral microstructural integrity and cognitive function. Neurology. (2010) 74:1022-9. doi: 10.1212/WNL.0b013e3181d7b45b

16. Altendahl M, Maillard P, Harvey D, Cotter D, Walters S, Wolf A, et al. An IL-18-centered inflammatory network as a biomarker for cerebral white matter injury. PLoS ONE. (2020) 15:e0227835. doi: 10.1371/journal.pone. 0227835

17. Walker KA, Power MC, Hoogeveen RC, Folsom AR, Ballantyne CM, Knopman DS, et al. Midlife systemic inflammation, latelife white matter integrity, and cerebral small vessel disease: the atherosclerosis risk in communities study. Stroke. (2017) 48:3196-202. doi: 10.1161/STROKEAHA.117.018675

18. Cai A, Zheng D, Qiu R, Mai W, Zhou Y. Lipoprotein-associated phospholipase A2 (Lp-PLA): a novel and promising biomarker for cardiovascular risks assessment. Dis Markers. (2013) 34:323-31. doi: 10.1155/2013/432136

19. Romero JR, Preis SR, Beiser AS, DeCarli C, Lee DY, Viswanathan $\mathrm{A}$, et al. Lipoprotein phospholipase A2 and cerebral microbleeds in the Framingham Heart Study. Stroke. (2012) 43:3091-4. doi: 10.1161/STROKEAHA.112.656744

20. Lee KO, Woo MH, Chung D, Choi JW, Kim NK, Kim OJ, et al. Differential impact of plasma homocysteine levels on the periventricular and subcortical white matter hyperintensities on the brain. Front Neurol. (2019) 10:1174. doi: 10.3389/fneur.2019.01174

21. Dhamoon MS, Cheung YK, Bagci A, Alperin N, Sacco RL, Elkind MSV, et al. Periventricular white matter hyperintensities and functional decline. J Am Geriatr Soc. (2018) 66:113-9. doi: 10.1111/jgs.15149

22. Jellinger PS, Smith DA, Mehta AE, Ganda O, Handelsman Y, Rodbard HW, et al. American association of clinical endocrinologists' guidelines for management of dyslipidemia and prevention of atherosclerosis. Endocr Pract. (2012) 18(Suppl 1):1-78. doi: 10.4158/EP.18.S1.1

23. Liu H, Yao Y, Wang Y, Ji L, Zhu K, Hu H, et al. Association between high-sensitivity $\mathrm{C}$-reactive protein, lipoprotein-associated phospholipase A2 and carotid atherosclerosis: a cross-sectional study. J Cell Mol Med. (2018) 22:5145-50. doi: 10.1111/jcmm. 13803

24. Fazekas F, Chawluk JB, Alavi A, Hurtig HI, Zimmerman RA. MR signal abnormalities at $1.5 \mathrm{~T}$ in Alzheimer's dementia and normal aging. AJR Am J Roentgenol. (1987) 149:351-6. doi: 10.2214/ajr.149.2.351

25. Zhu S, Wei X, Yang X, Huang Z, Chang Z, Xie F, et al. Plasma Lipoprotein-associated phospholipase $\mathrm{A} 2$ and superoxide dismutase are independent predicators of cognitive impairment in cerebral small vessel disease patients: diagnosis and assessment. Aging Dis. (2019) 10:834-46. doi: 10.14336/AD.2019.0304 
26. Wright CB, Moon Y, Paik MC, Brown TR, Rabbani L, Yoshita M, et al. Inflammatory biomarkers of vascular risk as correlates of leukoariosis. Stroke. (2009) 40:3466-71. doi: 10.1161/STROKEAHA.109.559567

27. Wardlaw JM, Smith C, Dichgans M. Small vessel disease: mechanisms and clinical implications. Lancet Neurol. (2019) 18:684-96. doi: 10.1016/S1474-4422(19)30079-1

28. Low A, Mak E, Rowe JB, Markus HS, O’Brien JT. Inflammation and cerebral small vessel disease: a systematic review. Ageing Res Rev. (2019) 53:100916. doi: 10.1016/j.arr.2019.100916

29. Zhou F, Liu Y, Shi H, Huang Q, Zhou J. Relation between lipoproteinassociated phospholipase A2 mass and incident ischemic stroke severity. Neurol Sci. (2018) 39:1591-6. doi: 10.1007/s10072-018-3474-3

30. van Oijen M, van der Meer IM, Hofman A, Witteman JC, Koudstaal PJ, Breteler MM. Lipoprotein-associated phospholipase A2 is associated with risk of dementia. Ann Neurol. (2006) 59:139-44. doi: 10.1002/ana.20721

31. Esenwa CC, Elkind MS. Inflammatory risk factors, biomarkers and associated therapy in ischaemic stroke. Nat Rev Neurol. (2016) 12:594-604. doi: 10.1038/nrneurol.2016.125

32. Shoamanesh A, Preis SR, Beiser AS, Vasan RS, Benjamin EJ, Kase $\mathrm{CS}$, et al. Inflammatory biomarkers, cerebral microbleeds, and small vessel disease: Framingham Heart Study. Neurology. (2015) 84:825-32. doi: 10.1212/WNL.0000000000001279

33. Huang F, Wang K, Shen J. Lipoprotein-associated phospholipase A2: the story continues. Med Res Rev. (2020) 40:79-134. doi: 10.1002/med.21597

34. Fernando MS, Simpson JE, Matthews F, Brayne C, Lewis CE, Barber R, et al. White matter lesions in an unselected cohort of the elderly: molecular pathology suggests origin from chronic hypoperfusion injury. Stroke. (2006) 37:1391-8. doi: 10.1161/01.STR.0000221308.94473.14
35. Fazekas F, Kleinert R, Offenbacher H, Schmidt R, Kleinert G, Payer F, et al. Pathologic correlates of incidental MRI white matter signal hyperintensities. Neurology. (1993) 43:1683-9. doi: 10.1212/WNL.43.9.1683

36. Kim KW, MacFall JR, Payne ME. Classification of white matter lesions on magnetic resonance imaging in elderly persons. Biol Psychiatry. (2008) 64:273-80. doi: 10.1016/j.biopsych.2008.03.024

37. Wardlaw JM, Smith C, Dichgans M. Mechanisms of sporadic cerebral small vessel disease: insights from neuroimaging. Lancet Neurol. (2013) 12:483-97. doi: 10.1016/S1474-4422(13)70060-7

38. Schmidt R, Schmidt H, Haybaeck J, Loitfelder M, Weis S, Cavalieri M, et al. Heterogeneity in age-related white matter changes. Acta Neuropathol. (2011) 122:171-85. doi: 10.1007/s00401-011-0851-X

39. Jiang JY, Gao YY, Zhang R, Wang L, Zhao X, Dai Q, et al. Differential effects of serum lipoprotein-associated phospholipase A2 on periventricular and deep subcortical white matter hyperintensity in brain. Research Square. (2020). doi: $10.21203 /$ rs.3.rs-44790/v1

Conflict of Interest: The authors declare that the research was conducted in the absence of any commercial or financial relationships that could be construed as a potential conflict of interest.

Copyright (c) 2021 Jiang, Gao, Zhang, Wang, Zhao, Dai, Zhang, Xu and Chen. This is an open-access article distributed under the terms of the Creative Commons Attribution License (CC BY). The use, distribution or reproduction in other forums is permitted, provided the original author(s) and the copyright owner(s) are credited and that the original publication in this journal is cited, in accordance with accepted academic practice. No use, distribution or reproduction is permitted which does not comply with these terms. 